

\title{
Ondes acoustiques sur l'arête d'un solide
}

\author{
W. Pajewski, M. Szalewski, P. Kielczyński
}

\section{To cite this version:}

W. Pajewski, M. Szalewski, P. Kielczyński. Ondes acoustiques sur l'arête d'un solide. Revue de Physique Appliquée, 1987, 22 (2), pp.113-118. 10.1051/rphysap:01987002202011300 . jpa-00245522

\section{HAL Id: jpa-00245522 https://hal.science/jpa-00245522}

Submitted on 1 Jan 1987

HAL is a multi-disciplinary open access archive for the deposit and dissemination of scientific research documents, whether they are published or not. The documents may come from teaching and research institutions in France or abroad, or from public or private research centers.
L'archive ouverte pluridisciplinaire HAL, est destinée au dépôt et à la diffusion de documents scientifiques de niveau recherche, publiés ou non, émanant des établissements d'enseignement et de recherche français ou étrangers, des laboratoires publics ou privés. 
Classification

Physics Abstracts

43.20M - 43.35P

\title{
Ondes acoustiques sur l'arête d'un solide
}

\author{
W. Pajewski, M. Szalewski et P. Kiełczyński \\ Laboratoire d'Acoustoélectronique, Institut des Problèmes Fondamentaux Techniques, Académie Polonaise \\ des Sciences, 00-049 Varsovie, ul. Swiętokrzyska 21, Pologne
}

(Reçu le 7 avril 1986, révisé le 19 septembre, accepté le 24 octobre 1986)

\begin{abstract}
Résumé. - Pour les solides limités, des ondes se propageant sur des arêtes existent, outre les ondes de volume et les ondes de surface. On appelle ces ondes - les ondes d'arête ou les ondes de ligne. Dans ce travail les résultats des études expérimentales sur la propagation d'ondes d'arête sur échantillons de verre, céramique $\mathrm{PZT} \mathrm{SiO}_{2}$ et $\mathrm{LiNbO}_{3}$ sont présentés. L'interaction non linéaire de deux ondes d'arête qui se propagent en sens opposés a été étudiée pour les échantillons de PZT et $\mathrm{LiNbO}_{3}$. Une convolution acoustique a été obtenue. On a discuté également le procédé de réalisation des guides. Aussi deux nouvelles méthodes d'excitation d'ondes d'arête sont proposées.
\end{abstract}

Abstract. - Acoustic waves propagating along edges formed by two surfaces exist in limited solids in addition to bulk and surface waves. These waves are called edge or line waves. Propagation of the edge waves has been investigated experimentally on samples of glass, PZT ceramics, $\mathrm{SiO}_{2}$ and $\mathrm{LiNbO}_{3}$. Edge wave convolvers have been fabricated and experimentally investigated using PZT and $\mathrm{LiNbO}_{3}$ samples. In the paper, methods of waveguides fabrication are discussed. Some new possibilities of edge waves excitation are also proposed.

\section{Introduction.}

Pour les solides limités, des ondes se propageant sur des arêtes existent, outre les ondes de volume et les ondes de surface. On appelle ces ondes - les ondes d'arête ou les ondes de ligne. Elles comprennent trois composantes du mouvement des particules. Leur amplitude diminue en fonction de la distance de l'arête sur les deux plans ainsi qu'à l'intérieur du solide. Sur un solide isotrope deux types fondamentaux d'ondes d'arête existent sous différentes formes de déformation. Pour un solide anisotrope la forme de la déformation de l'arête pendant la propagation des ondes est plus compliquée : les modes purs n'existent pas.

Dans les guides d'ondes d'arête, de grandes densités de puissance se manifestent puisque la puissance acoustique se concentre dans le voisinage de l'arête, dans un espace d'une section plus petite que $\lambda^{2}$ ( $\lambda$ est la longueur d'onde d'arête). Pour $\mathrm{LiNbO}_{3}$ on peut obtenir des densités de puissance jusqu'à $600 \mathrm{~W} / \mathrm{mm}^{2}$, pour une fréquence d'environ $200 \mathrm{MHz}$ [1]. Une grande densité de puissance favorise l'obtention d'effets non linéaires : convolution acoustique et effets paramétriques.

L'obtention d'une arête suffisamment acérée présente la plus grande difficulté pour la réalisation de ces guides. Un arrondissement de l'arête doit être d'un ordre de grandeur inférieur à la longueur de l'onde. Ces difficultés limitent le domaine de fréquences dans lequel on peut utiliser des guides d'ondes d'arête. Pour des fréquences jusqu'à $200 \mathrm{MHz}$, on peut réaliser des arêtes acérées en profitant du clivage de cristaux. Les défauts de cette technologie sont : la difficulté d'obtention des échantillons longs de bonne qualité et l'impossibilité de choisir l'orientation d'un cristal. Une coupe effectuée en utilisant une scie de diamant permet d'obtenir des arêtes pour des fréquences jusqu'à $100 \mathrm{MHz}$ [1].

\section{Méthodes d'excitation des ondes d'arête.}

On peut utiliser, en pratique, plusieurs méthodes d'excitation des ondes d'arête. Pour cela nous avons employé des transducteurs piézoélectriques, sous forme de plaques, placés à l'arête directement sur un guide d'ondes (Fig. 1a). Le type d'onde, que ces transducteurs excitent, dépend de leur positionnement par rapport au plan de symétrie de l'angle dièdre. Une autre méthode d'excitation d'onde d'arête, reportée dans [2], consiste à employer un transducteur interdigital monophase déposé sur un des plans dans le voisinage de l'arête. Dans ce cas un couplage électromécanique convenable doit exister. On peut améliorer cette méthode d'excitation en utilisant un coupleur à réseau. Celui-ci permet d'obtenir l'adaptation de phase de l'onde de Rayleigh à l'onde d'arête qui possède une vitesse plus petite que celle de l'onde de Rayleigh [2]. 


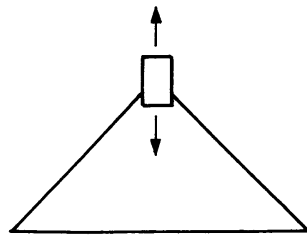

$\Gamma_{1}$

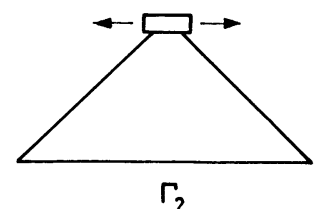

Fig. 1a. - Excitation d'une onde d'arête à l'aide d'un transducteur en forme de plaque.

[Edge waves generation by plate transducers.]

Il est possible aussi d'utiliser la transformation de l'onde de Rayleigh en onde d'arête. L'onde de Rayleigh se propageant sur la surface se réfléchit partiellement à l'arête, partiellement elle traverse vers l'autre surface ; le reste d'énergie se transforme en onde de volume et en onde d'arête. La distribution d'énergie entre les ondes particulières dépend de l'angle d'incidence de l'onde de Rayleigh. La qualité de l'arête exerce aussi une influence essentielle, un arrondissement de l'arête cause une augmentation de l'amplitude de l'onde de Rayleigh traversante. On a vérifié expérimentalement que pour le verre, pour l'angle d'incidence de $75^{\circ}$, presque $60 \%$ d'énergie de l'onde de Rayleigh se transforme en onde d'arête. Des mesures ont été effectuées à l'aide de transducteurs piézoélectriques, sous forme de plaques, placés sur un demi-cylindre de verre (Fig. 1b). On a obtenu aussi la transformation de l'onde de Rayleigh en onde d'arête pour les échantillons de quartz, coupe $X$, une arête orientée selon l'axe $Y$. Cette méthode d'excitation d'ondes d'arête n'a pas été publiée précédemment.

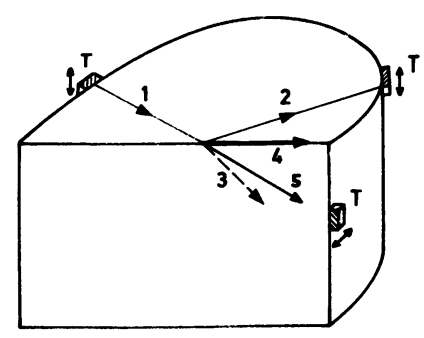

Fig. 1b. - Transformation de l'onde de Rayleigh en onde d'arête sur un échantillon rectangulaire. Solide isotrope. 1 onde de Rayleigh incidente, 2 - onde de Rayleigh réfléchie, 3 - onde de volume diffractée, 4 - onde d'arête engendrée, 5 onde de Rayleigh transmise, $\mathrm{T}$ - transducteur.

[Rayleigh wave transformation into edge wave in rectangular sample of isotropic solid. 1 - incident Rayleigh wave, 2reflected Rayleigh wave, 3 - diffracted volume wave, 4 - edge wave, 5 - transmitted Rayleigh wave, $\mathrm{T}$ - transducer.]

\section{Ondes d'arête sur un solide isotrope.}

Sur un solide isotrope deux types fondamentaux d'ondes d'arête existent - le mode symétrique $\Gamma_{1}$ et le mode antisymétrique $\Gamma_{2}$. Pour le mode $\Gamma_{1}$ les déforma- tions sur l'arête sont étendues sur le plan de symétrie de l'angle dièdre : les déformations sur les plans formant cet angle sont symétriques (Fig. 2). Pour le mode $\Gamma_{2}$ les déformations sur l'arête sont perpendiculaires au plan de symétrie et les déformations sur les plans formant l'angle sont antisymétriques à l'égard du plan de symétrie de l'angle dièdre (Fig. 2b). Le mode $\Gamma_{1}$ est dispersif, le mode $\Gamma_{2}-$ non dispersif. Les axes $x_{1}, x_{2}, x_{3}$ sur les figures sont les axes d'un système de coordonnées. Pour un solide isotrope le choix des axes est complètement arbitraire.

On a étudié expérimentalement la propagation d'ondes sur les arêtes d'échantillons de verre. La figure 3a montre les impulsions de l'onde $\Gamma_{2}$ sur un échantillon de verre, à la fréquence de $4 \mathrm{MHz}$, utilisant un transducteur à plaque de céramique PZT. Une surcharge mécanique de l'arête (p. ex. une pression sur l'arête, des surfaces latérales libres) provoquera l'atténuation d'un signal (Fig. 3b). Cet effet confirme que l'onde se propageant n'est pas l'onde de volume ou l'onde de Rayleigh mais effectivement l'onde de l'arête.
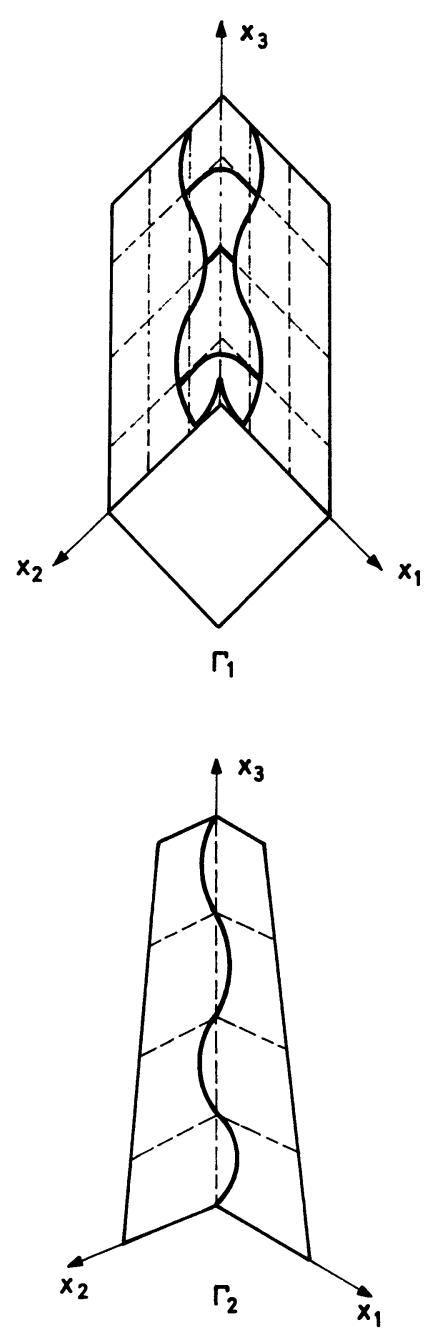

b)

Fig. 2. - a) Onde d'arête $\Gamma_{1}$. b) Onde d'arête $\Gamma_{2}$. [Edge wave $\Gamma_{1}$. Edge wave $\Gamma_{2}$.] 


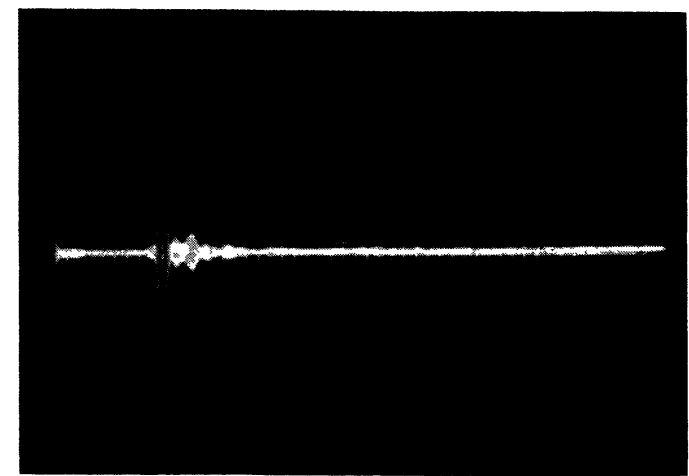

a)

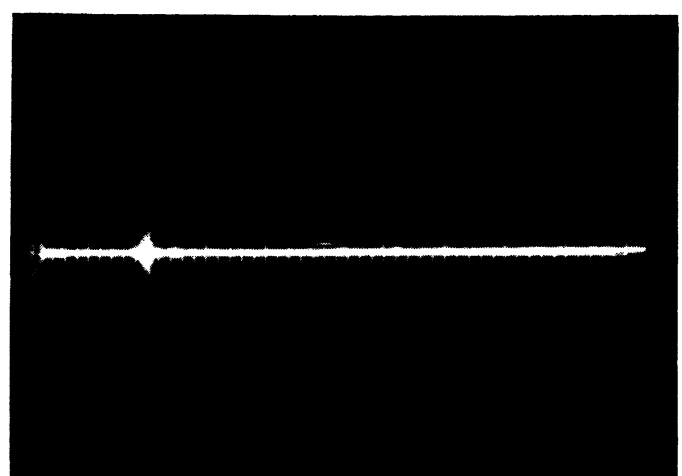

b)

Fig. 3. - Impulsion de l'onde $\Gamma_{2}$ sur un échantillon de verre.

[ $\Gamma_{2}$ wave on edge of glass sample.]

Les deux modes peuvent se propager sur l'arête courbée. La figure 4 montre les impulsions de l'onde $\Gamma_{2}$ se propageant sur l'arête d'un demi-cylindre de verre de rayon $30 \mathrm{~mm}$.

\section{Ondes d'arête sur un solide anisotrope.}

En raison de l'anisotropie, les ondes d'arête sont seulement plus ou moins proches du type $\Gamma_{1}$ ou $\Gamma_{2}$, selon que la déformation domine : perpendiculaires au plan de symétrie ou parallèles.

Nous avons étudié des barres de quartz orientées selon l'axe $x$, avec les plans $y$ et $z$ et des barres orientées selon l'axe $y$, avec les plans $x$ et $z$. Les calculs numériques réalisés ont permis de trouver les distributions des composantes de vibrations des particules sur les plans $y$ et $z$, ou $x$ et $z$. Les calculs numériques furent réalisés à l'aide de l'ordinateur Cyber CDC en utilisant les équations analytiques présentées déjà dans [3,4]. A cette occasion un programme en Fortran a été préparé [5].

La figure 5 montre les amplitudes relatives des composantes des vibrations en fonction de la distance de l'arête orientée selon l'axe $x$, pour deux directions $y$ et $z$, où $x, y, z$ correspondent aux axes cristallographiques du quartz. Le mode proche du mode $\Gamma_{2}$ est aussi excité pour ces barres.

Nous avons aussi effectué des calculs et des mesures pour le quartz de coupes $y$ et ST. La figure 6 montre la distribution calculée des vibrations pour le quartz de coupe ST.

Les ondes d'arête sur les échantillons de quartz de coupes $x, y$ et ST ont été excitées à l'aide de transducteurs à plaque de quartz $y$. Les oscillogrammes exemplaires des impulsions sont présentés sur les figures 7 et 8.

Nous avons aussi étudié des échantillons de niobate de lithium et de céramique piézoélectrique du type PZT. Deux orientations de $\mathrm{LiNbO}_{3}$ ont été utilisées : l'arête obtenue du plan du clivage et du plan de normale $y-128^{\circ}$ et l'arête d'intersection des plans $x$ et $y$, direction de propagation $z$. Des ondes d'arête étaient excitées à l'aide de transducteurs de $\mathrm{LiNbO}_{3}$, coupe $y-163^{\circ}$. On a obtenu des ondes de fréquence $70 \mathrm{MHz}$ en travaillant sur le $7^{\mathrm{e}}$ harmonique.


Fig. 4. - Impulsion d'onde $\Gamma_{2}$ se propageant sur l'arête d'un demi-cylindre de verre, fréquence $4 \mathrm{MHz}$, à droite avec une pression sur l'arête.

[ $\Gamma_{2}$ wave on edge of glass semicylinder, frequency $4 \mathrm{MHz}$, on the right with pressure on the edge.] 

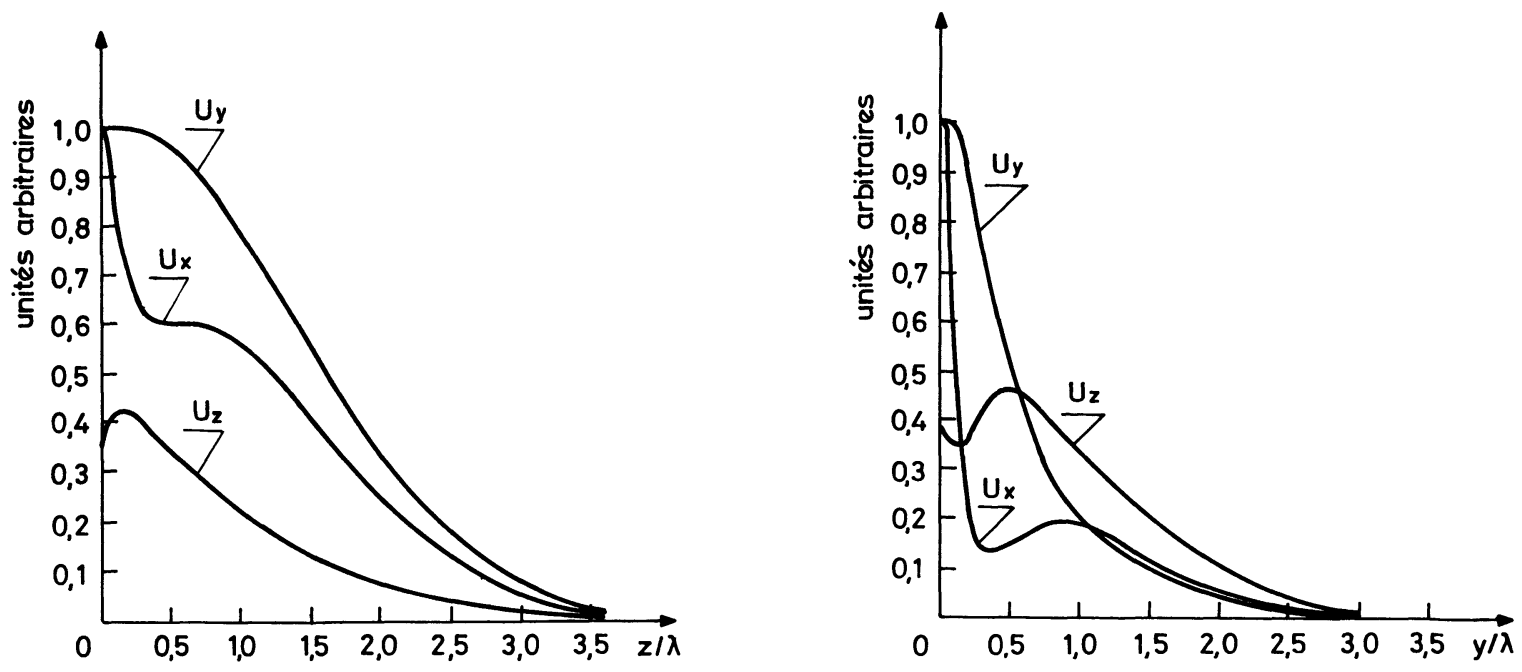

Fig. 5. - Amplitudes relatives des composantes $x, y, z$ de l'onde d'arête sur la barre de quartz de coupe $x$.

[Relative amplitudes of $x, y, z$ components of edge wave, quartz bar of $x$ cut.]



Fig. 6. - Distribution des vibrations pour l'onde d'arête sur la barre de quartz de coupe ST.

[Distribution of vibrations for edge wave on ST cut quartz bar.]

\section{Convolution acoustique.}

La convolution acoustique est utilisée pour le traitement du signal. Le signal de sortie pour deux signaux d'entrée $V_{1}(t)$ et $V_{2}(t)$ se propageant en sens opposés est égal à

$$
V_{3}(t)=A \int_{-\infty}^{+\infty} V_{1}(\tau) V_{2}(2 t-\tau) \mathrm{d} \tau
$$

Lorsque les signaux $V_{1}(t)$ et $V_{2}(t)$ sont des impulsions rectangulaires identiques avec une fréquence porteuse $f_{1}$, on obtient à la sortie une impulsion triangulaire avec une fréquence porteuse $2 f_{1}$. La tension de sortie est proportionnelle à la durée des impulsions d'entrée et à la puissance acoustique par unité de largeur du faisceau d'onde [6].

L'interaction non linéaire de deux ondes d'arête
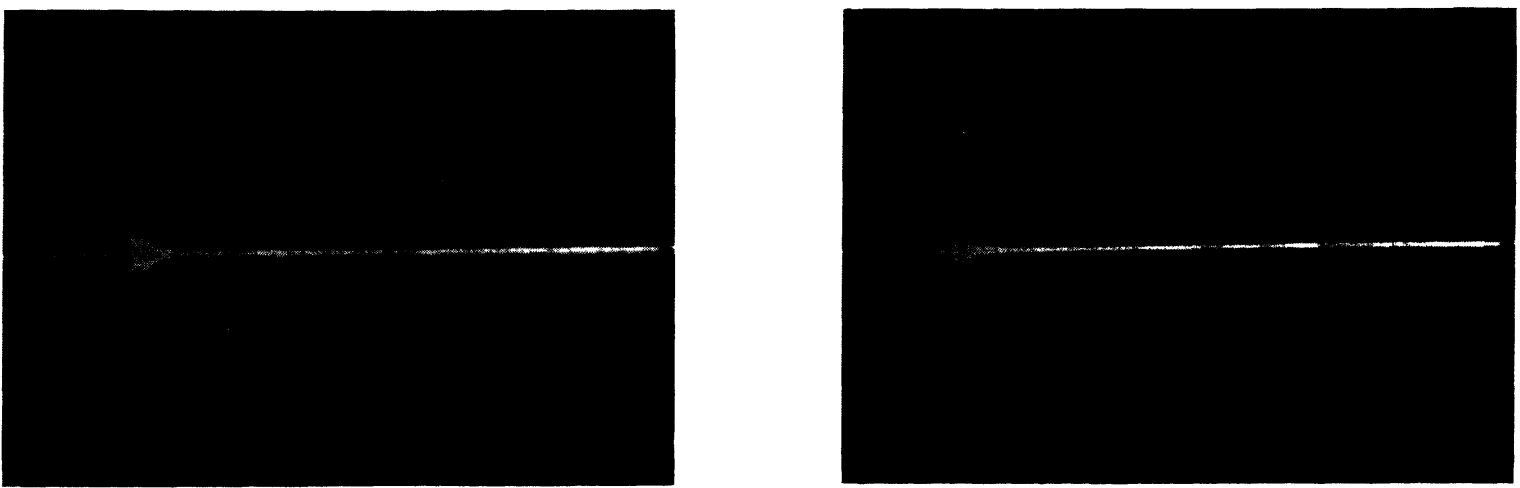

Fig. 7. - Impulsion de l'onde d'arête proche de $\Gamma_{2}$ sur un échantillon de quartz de coupe $x$, fréquence $4 \mathrm{MHz}$, à droite avec une pression à l'arête.

[Edge wave similar to $\Gamma_{2}$ mode on $x$ cut quartz sample, frequency $4 \mathrm{MHz}$, on the right with pressure on the edge.] 

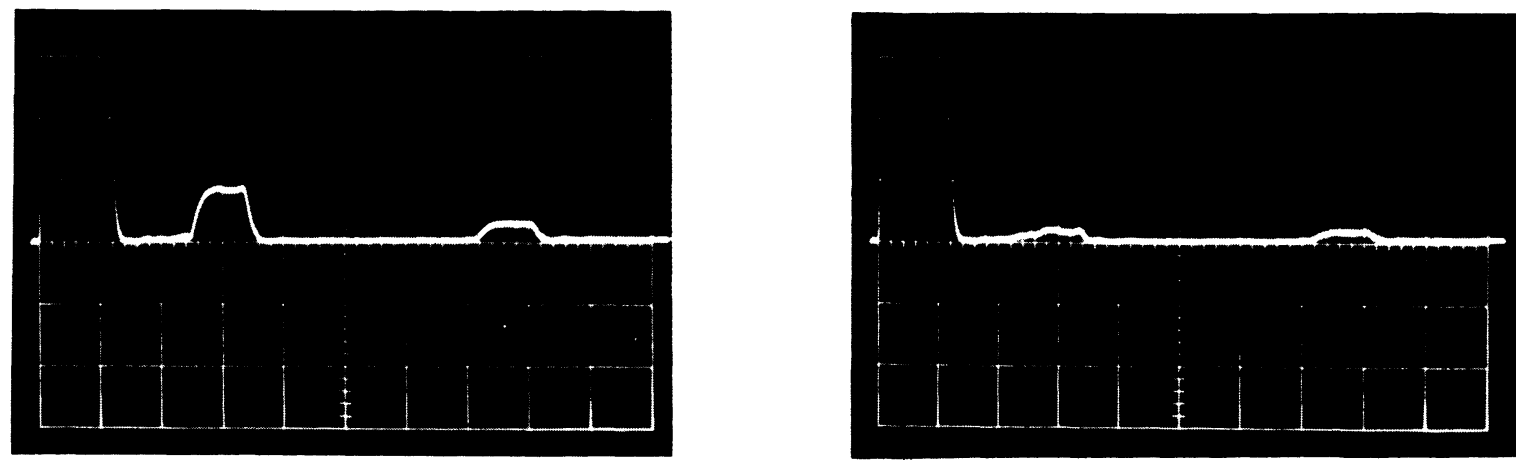

Fig. 8. - Impulsion de l'onde d'arête sur un échantillon de quartz de coupe ST, fréquence $31 \mathrm{MHz}$, à droite avec une pression sur l'arête, $x_{1}$ : distance de l'arête sur la surface ST.

[Edge wave on ST cut quartz sample, frequency $31 \mathrm{MHz}$, on the right with pressure on the edge, $x_{1}$ : distance from the edge on the ST surface.]

$\Gamma_{2}$ se propageant en sens opposés suscite la formation d'un potentiel superficiel et symétrique par rapport à la bissectrice de l'angle dièdre [4]. Pour une telle distribution de potentiel, on ne peut pas utiliser des électrodes de détection situées symétriquement par rapport à l'arête. Une électrode de détection doit être éloignée de l'arête d'une distance d'environ $\lambda$ et la deuxième surface doit être entièrement métallisée.

Des dispositifs de convolution ont été réalisés sur la céramique PZT et sur $\mathrm{LiNbO}_{3}$ (orientation comme cidessus). Les électrodes de détection étaient situées sur le plan $y-128^{\circ}$ ou sur le plan $y$. Une convolution acoustique a été obtenue pour les impulsions simples et doubles [7]. La figure 9 montre les signaux de convolu-

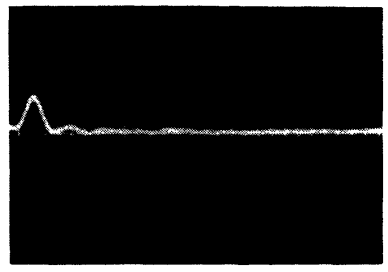

a)

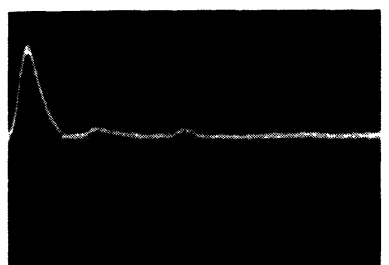

c)

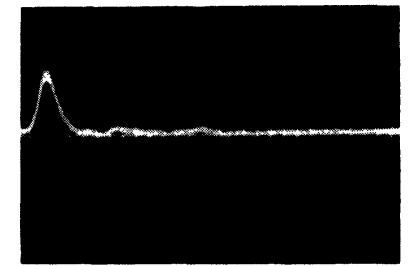

b)

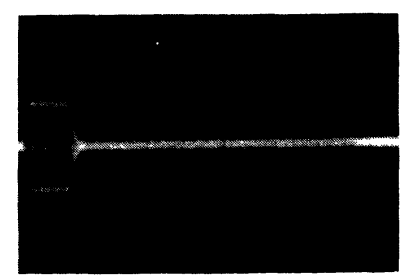

d) tion pour l'onde d'arête sur la céramique PZT, à une fréquence porteuse des signaux d'entrée de $8 \mathrm{MHz}$ et une fréquence porteuse du signal de sortie à $16 \mathrm{MHz}$. L'agrandissement de la durée des impulsions d'entrée produit l'agrandissement de l'amplitude du signal de convolution. Le signal de sortie disparaît en présence d'une pression sur l'arête ou après le débranchement d'un des transducteurs-émetteurs. Cet effet confirme que le signal reçu se produit à la suite de l'interaction de deux ondes d'arête se propageant en sens opposé. Des expériences ont été conduites pour les fréquences porteuses des signaux d'entrée 8-12,5 MHz. L'augmentation de fréquences au-dessus de $100 \mathrm{MHz}$ rendrait possible une beaucoup plus forte concentration d'onde. Cela entraînerait un agrandissement considérable du signal de sortie pour la même puissance d'entrée.

L'application des ondes d'arête permet d'obtenir, après l'optimisation du dispositif de convolution, une efficacité comparable avec celle obtenue dans des dispositifs de type piézoélectrique-semiconducteur. L'avantage additionnel des ondes d'arête semble être l'absence de dispersion (mode $\Gamma_{2}$ ) et de diffraction, qui mènent à des déformations de signal dans les convoluteurs avec les ondes de Rayleigh.

\section{Conclusion.}

Les ondes d'arête sont utilisées dans les dispositifs de traitement de signal et dans les dispositifs acoustoélectriques à semiconducteur [8]. La technologie de réalisation d'arêtes de qualité convenable et les méthodes d'excitation efficaces d'ondes déterminent les possibilités d'applications pratiques. Comme nos expériences l'ont montré, l'utilisation de transducteurs sous forme de plaques constitue une méthode simple et efficace d'excitation des ondes d'arête pour les fréquences de quelques $\mathrm{MHz}$ à quelques dizaines de $\mathrm{MHz}$. Pour des fréquences plus élevées il faut utiliser des transducteurs interdigités [2]. Cependant, la réalisation de ces structu-

Fig. 9. - Signaux de convolution pour l'onde d'arête sur céramique PZT, durée de l'impulsion d'entrée : a) $3 \mu \mathrm{s}$, b) 3,5 $\mu \mathrm{s}$, c) $4 \mu \mathrm{s}$, d) forme de l'impulsion d'entrée.

[Convolution signals for edge wave on PZT ceramics, input signals width : a) $3 \mu \mathrm{s}$, b) $3.5 \mu \mathrm{s}$, c) $4 \mu \mathrm{s}$, d) shape of input signal.] 
res, avec la distance de l'arête égale à environ $\lambda / 3$, crée des problèmes technologiques considérables. Les ondes d'arête peuvent être également excitées, avec une grande efficacité $(60 \%)$, en utilisant les ondes de Rayleigh à incidence oblique sur une arête. Dans ce cas les études théoriques restent à faire.

\section{Remerciements.}

Les auteurs remercient Monsieur Joost Horsten de Technische Hogenschool Eindhoven pour sa collaboration à l'analyse numérique, lors de son stage dans notre institut.

\section{Bibliographie}

[1] Hoskins, M. J., Datta, S., Panasik, C. M., HunsinGER, B. J., Line acoustic waves on cleaved edges in $\mathrm{LiNbO}_{3}$ and $\mathrm{GaAs}, 1978$, IEEE Ultrasonics Symp. Proc.

[2] Miller, R. L., Pieters, G. C., Hunsinger, B. J., Broadband generation of line acoustic waves, IEEE Trans. Sonics Ultrason. SU-32 (1985) 440449.

[3] Maradudin, A. A., Wallis, R. F., Mills, D. L., BALLARD, R. L., "Vibrational edge modes in finite crystals », Phys. Rev. B 6, 4 (1972) 11061111.

[4] Datta, S., Hunsinger, B. J., « Analysis of line acoustic wave in general piezoelectric crystals », Phys. Rev. B 16, 10 (1977) 4224-4229.
[5] Horsten, J., Numerical analysis of waves on edges of piezoelectric crystal, Internal Report, Eindhoven University of Technology, Faculty of Physical Engineering (1984).

[6] Bridoux, E., Rouvaen, J. M., Delaunoy, M., MORIAMEZ, M., Interaction non linéaire de deux ondes acoustiques superficielles se propageant en sens opposés à la surface d'un cristal piézoélectrique, J. Physique 33 (1972) 245-251.

[7] Pajewski, W., Szalewski, M., « Konwolucja na falach krawędziowych », Elektron. 26, $\mathrm{n}^{\text {os }}$ 11-12 (1985) 9-11 (en polonais).

[8] Hoskins, M., Fliegel, F., Mahon, S., Electron transport by line acoustic waves in $\mathrm{GaAs}: \mathrm{Cr}$, 1980 IEEE Ultrasonics Symp. Proc. 